\title{
Influence of HIV and other risk factors on tuberculosis
}

\author{
S Mahtab, ${ }^{1}$ MBBS, MPH; D Coetzee, ${ }^{2}$ FCPHM \\ ${ }^{1}$ Department of Paediatrics, Faculty of Health Sciences, University of Cape Town, South Africa \\ ${ }^{2}$ Centre for Infectious Disease and Epidemiology and Research, Faculty of Health Sciences, University of Cape Town, South Africa
}

Corresponding author: S Mahtab (sanamahtab@hotmail.com)

\begin{abstract}
Background. Tuberculosis (TB) notification in South Africa has increased six-fold over the past two decades, mainly because of the HIV epidemic. Objectives. To describe the sociodemographic and outcome characteristics of TB patients, and to identify risk factors associated with TB treatment outcomes stratified by HIV status.

Methods. A cross-sectional study was used to analyse data from the Cape Town Metro East geographical service area (GSA) electronic TB register (ETR.Net), including adult patients aged $\geq 15$ years who initiated TB treatment between 1 July 2011 and 30 June 2012.

Results. TB case notification in the GSA was 922 per 100000 population. Of the 12672 TB patients registered, 50.5\% were co-infected with HIV. The death rate in co-infected patients was $5.4 \%$ v. $2.8 \%$ in HIV-negative patients, the rate of treatment success $66.6 \%$ v. $73.5 \%$, and the rate of unfavourable treatment outcome $28.1 \%$ v. $23.7 \%$. The Khayelitsha subdistrict had the highest proportion of TB burden (37.0\%) and co-infection (47.6\%). Fourteen percent of patients had extrapulmonary TB, $65.9 \%$ of whom were co-infected with HIV. In the multivariate analysis, HIV infection (relative risk (RR) 1.2), retreatment (RR 1.4) and sputum smear microscopy not done (RR 1.4) were significantly associated with unfavourable treatment outcome. The Eastern (RR 0.9) and Northern (RR 0.7) subdistricts were less likely to have unfavourable outcomes compared with Khayelitsha. In the stratified analysis, retreatment and smear not done were significant risk factors for an unfavourable treatment outcome in both co-infected and HIV-negative patients.

Conclusions. The burdens of both TB and co-infection were high in this community, although HIV prevalence varied. Mortality was higher and treatment completion lower in co-infected patients than in those who were HIV-negative. Co-infection, previous TB treatment and smear not done were significant risk factors for an unfavourable outcome in all patients.
\end{abstract}

S Afr Med J 2017;107(5):428-434. DOI:10.7196/SAMJ.2017.v107i5.11271

The HIV epidemic in Africa has not only dramatically increased the incidence of tuberculosis (TB) in this region, but has also increased the TB mortality rate. Co-infected patients are also more likely to die from TB, which is more easily curable in HIV-negative patients. ${ }^{[1]}$ The rate of unfavourable treatment outcome for HIV-positive pulmonary TB patients in some African countries is now as high as $20 \%$ for sputum smear-positive cases and $50 \%$ for sputum smear-negative cases. $^{[2,3]}$ Deaths from TB represent a significant indicator of the severity of the effect of HIV on TB epidemiology. ${ }^{[1]}$ Extrapulmonary TB (EPTB), which is also a risk factor for unfavourable outcome, is 20 - 70\% more common in HIV-positive patients. ${ }^{[4]}$

In 2013, there were 1.5 million deaths from TB globally, 360000 of them associated with HIV ${ }^{[5]}$ Globally TB is the most common opportunistic infection in HIV-positive patients. Wood et al. ${ }^{[6]}$ reported a 17 times higher risk of developing TB disease in HIVpositive populations than in HIV-negative populations. TB has a negative impact on HIV, increasing the risk of HIV-related morbidity and mortality ${ }^{[7]}$ through the increased replication of HIV cell lines and activation of mononuclear cells, increasing the progression of HIV infection and susceptibility to other infections, and decreasing the time to development of AIDS. ${ }^{[8]}$

The increased mortality rate of TB patients in high HIV prevalence populations in Africa may change the general perception of TB as a curable disease and threaten the reputation of TB programmes. This may have an adverse influence on the willingness of individuals suspected of having TB to come forward for diagnosis and complete TB treatment. ${ }^{[1]}$

According to South African (SA) data, TB was the leading cause of death in persons 15 - 24 years of age in 2010, accounting for $14 \%$ of all deaths. ${ }^{[9]}$ In 2013, the World Health Organization (WHO) reported that $62 \%$ of TB patients in SA were co-infected with HIV. ${ }^{[10]}$ Co-infected patients have poorer treatment outcomes than patients who are HIV-negative. In 2011, the treatment success rate globally was $73 \%$ in co-infected patients and $87 \%$ in HIV-negative patients. ${ }^{[11]}$ In 2013, the incidence of TB in persons co-infected with HIV was 520 per 100000 in SA and mortality was 121 per 100000 , more than double the rate of 48 per 100000 in HIV-negative persons. ${ }^{[10]}$

\section{Objectives}

The primary objective of this study was to describe the sociodemographic and outcome characteristics of TB patients in the Metro East geographical service area (GSA), Cape Town, SA. The secondary objective was to identify risk factors associated with TB treatment outcomes stratified by HIV status.

\section{Methods}

Study design

A cross-sectional study was conducted using quantitative research methods on data from the electronic TB register (ETR.Net). ETR. Net is an electronic TB register that monitors all patients treated for drug-sensitive TB at public sector health facilities. The register is prospectively completed as the person progresses though their course of TB treatment. It contains the following information: date of treatment initiation, basic demographics, previous TB treatment, laboratory test results at initiation, at 2 or 3 months and at completion of treatment, and HIV status. In most facilities, this information is collected in a paper TB register and then transferred to ETR.Net at subdistrict level. This information is used to generate standard reports and provide data on the quality of services, so that the $\mathrm{TB}$ strategy and programme can be monitored and evaluated. 


\section{Study setting}

The district of Cape Town is divided into two GSAs, Metro East and Metro West. Data for the Metro East GSA were analysed in this project. The Metro East GSA consists of the Eastern, Khayelitsha, Northern and Tygerberg subdistricts.

\section{Population}

The study population included persons aged $\geq 15$ years, placed on TB treatment regimens 1 or 2 at public sector TB services in the Metro East GSA of Cape Town and registered on ETR.Net for TB treatment initiation from 1 July 2011 to 30 June 2012. The study only included individuals whose HIV status was known.

\section{Ethics approval}

Ethics approval was obtained from the Human Research Ethics Committee of the Faculty of Health Sciences at the University of Cape Town (UCT) (ref. no. 758/2014). The names and addresses of all participants were removed from the database before the study was conducted.

\section{TB treatment outcome}

The WHO classifies TB treatment outcomes into one of six categories: completed, cured, failed, died, defaulted and outcome unknown. ${ }^{[5]}$

Definitions:

- Cure. Patient smear- or culture-positive at the beginning of treatment and smear/culture-negative in the last month of treatment and on at least one previous occasion at least 30 days before that. ${ }^{[5]}$

- Treatment completed. Patient smear/culture-positive at the beginning and has completed treatment but did not have a negative smear/culture in the last month of treatment and on at least one previous occasion $>30$ days before that. The smear examination may not have been done or the results may not be available at the end of treatment. ${ }^{[5]}$

- Treatment success. Includes both treatment completion and cure. ${ }^{[5]}$

- Treatment default. Patient whose treatment was interrupted for 2 consecutive months or more during the treatment period. ${ }^{[5]}$

- Treatment failure. Smear/culture-positive patient who remains or is again smear-positive at 5 months or later during treatment. This definition also includes patients who are diagnosed with multidrug-resistant TB during treatment. ${ }^{[5]}$

- Treatment unsuccessful. Includes both treatment default and treatment failure.

- Died. Death for any reason during TB treatment. ${ }^{\left[{ }^{[5]}\right.}$

- Unfavourable treatment outcome. Includes treatment default, treatment failure and death.

\section{Data analysis}

All selected data were exported from Microsoft Excel version 2010 (Microsoft, USA) into STATA version 12.1 (StataCorp, USA) for analysis. Univariate analysis explored the distribution of the dependent and independent variables using frequency tables.

Bivariate analysis identified risk factors that were associated with HIV status and other risk factors and TB treatment outcomes. For the bivariate analysis, treatment default, treatment failure and death were merged into one variable, namely unfavourable treatment outcome. Pearson's $\chi^{2}$ statistic was used to compare categorical variables, and $p<0.05$ was considered statistically significant.

Relative risks (RRs) were used for measures of association. Univariate and multivariate analyses were performed using a generalised linear regression model. Statistically significant variables in the univariate analysis were included in the multivariate analysis.
The contribution of each variable to the model was evaluated using likelihood ratio $\chi^{2}$ tests

\section{Results}

Between 1 July 2011 and 30 June 2012, 14585 patients aged $\geq 15$ years were initiated on TB treatment and registered on the TB ETR.Net in the Metro East GSA. The study sample comprised 12672 patients after 1913 were excluded because they were not on treatment regimen 1 or 2 , their HIV status was not known, or they had been transferred into or out of the district (Fig. 1).

Table 1 presents the demographic characteristics, treatment category, site of TB, subdistrict where treated and diagnostic sputum smear microscopy results. Of patients initiated on TB treatment from 1 July 2011 to 30 June 2012, 77.4\% were between 15 and 44 years of age. The majority were males (54.5\%). The subdistrict with the highest proportion of the TB cases in the GSA was Khayelitsha (37.0\%) and the lowest was Northern (14.9\%). Of TB patients, 50.5\% were co-infected with HIV. Retreatment cases accounted for $30.4 \%$ of all cases, $83.4 \%$ of cases were classified as pulmonary TB, $88.8 \%$ of the patients had a diagnostic sputum smear microscopy result, and $46.5 \%$ of the patients had a positive sputum smear result.

Of HIV co-infected patients, $56.1 \%$ were aged 30 - 44 years, $53.3 \%$ were female, $47.6 \%$ were from Khayelitsha, $28.1 \%$ had an unsuccessful treatment outcome and 5.3\% died (Table 2). More HIV co-infected than HIV-negative patients were under retreatment $(33.2 \%$ v. $27.6 \%$, respectively), and more had $\mathrm{EPTB}$ and both pulmonary $\mathrm{TB}$ and $\mathrm{EPTB}$ ( $18.6 \%$ v. $9.8 \%$ and $3.5 \%$ v. $1.4 \%$, respectively).

Of all TB patients in the GSA, $70.0 \%$ had a successful treatment outcome, $25.9 \%$ had unsuccessful treatment and $4.1 \%$ died; $66.1 \%$ of patients who died and $54.7 \%$ of those who had unsuccessful treatment were co-infected, whereas $48.0 \%$ of those with a successful treatment success outcome were co-infected. Khayelitsha was the district with the highest proportion of unsuccessful outcomes (43.4\%) and deaths (38.7\%) (Table 3).

\section{TB case notification among districts}

Based on the population census of 2011, ${ }^{[12]}$ the TB case notification rate calculated for the Metro East GSA was 922 per 100 000. Rates of TB case notification and proportions of TB patients with HIV co-infection for each subdistrict are presented in Table 4, which also includes the annual HIV seroprevalence rates from the 2012 antenatal survey. ${ }^{[13]}$ The trend for HIV co-infection across subdistricts matches the seroprevalence of HIV in the antenatal survey.

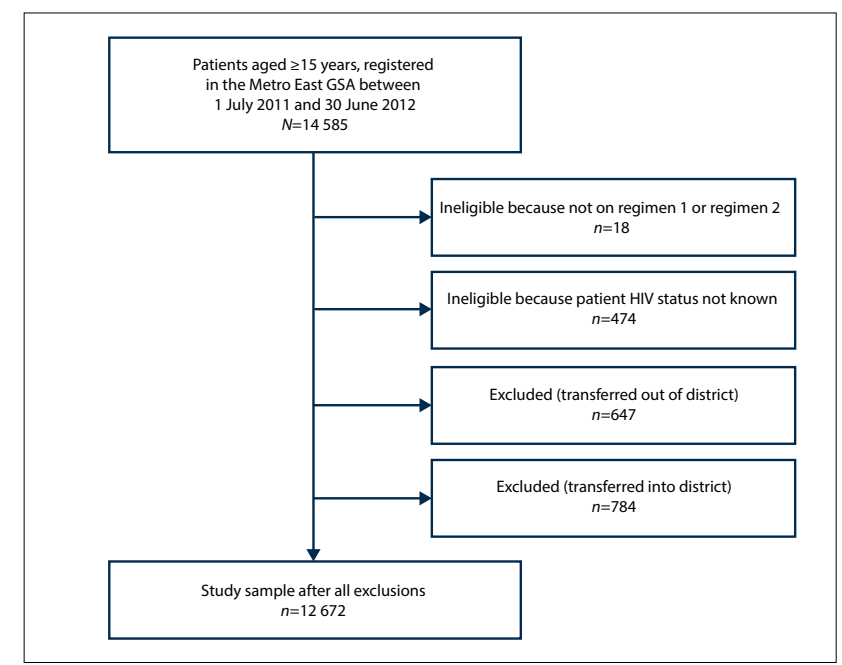

Fig. 1. Selection of the population included in the study. 


\begin{tabular}{ll} 
Table 1. Demographic characteristics, HIV status, treatment \\
category, site of TB, diagnostic sputum smear microscopy \\
results and treatment outcome of patients $(\boldsymbol{N}=\mathbf{1 2}$ 672) initia- \\
ted on TB treatment in the Metro East GSA, Cape Town \\
\hline Variables & $\boldsymbol{n}(\%)$ \\
\hline Age (years) & \\
$15-29$ & $4360(34.4)$ \\
$30-44$ & $5443(43.0)$ \\
$45-59$ & $2372(18.7)$ \\
$>59$ & $497(3.9)$ \\
Sex & $5766(45.5)$ \\
Female & $6906(54.5)$ \\
Male & \\
Subdistrict & $4683(37.0)$ \\
Khayelitsha & $2941(23.2)$ \\
Eastern & $1892(14.9)$ \\
Northern & $3156(24.9)$ \\
Tygerberg & \\
HIV status & $6275(49.5)$ \\
Negative & $6397(50.5)$ \\
Positive &
\end{tabular}

\section{Sputum smear conversion rates at the end of the intensive phase and at the end of treatment, stratified by HIV status}

In total, 11255 patients had a diagnostic sputum smear sent to the laboratory for microscopy and 5889 (52.3\%) of these were positive; of patients with a positive sputum smear, 2063 (35.0\%) were HIVpositive and $3826(65.0 \%)$ were HIV-negative.

Fig. 2 (A) shows that at the end of the intensive phase, 1707 (82.7\%) of HIV-positive patients who had a positive sputum smear at initiation had a negative smear, $88(4.3 \%)$ remained positive and 311 (15.1\%) did not have a smear. At the end of treatment, 14 smears (0.7\%) remained positive, but 703 patients $(34.1 \%)$ did not have a smear. In terms of outcome, 102 (14.5\%) of the 703 patients who did not have a smear died, 55 (7.8\%) had a successful outcome and 546 (77.7\%) had an unsuccessful outcome.

Fig. 2 (B) shows that at the end of the intensive phase, 3331 (87.1\%) of HIV-negative patients who had a positive sputum smear at initiation had a negative smear, 265 (6.9\%) remained positive and $375(9.8 \%)$ did not have a smear. At the end of treatment, 25 smears (0.7\%) remained positive but 1003 (26.2\%) did not have a smear. In terms of outcome, $66(6.6 \%)$ of the 1003 patients who did not have a smear died, $84(8.4 \%)$ had a successful outcome and $853(85.1 \%)$ had an unsuccessful outcome.

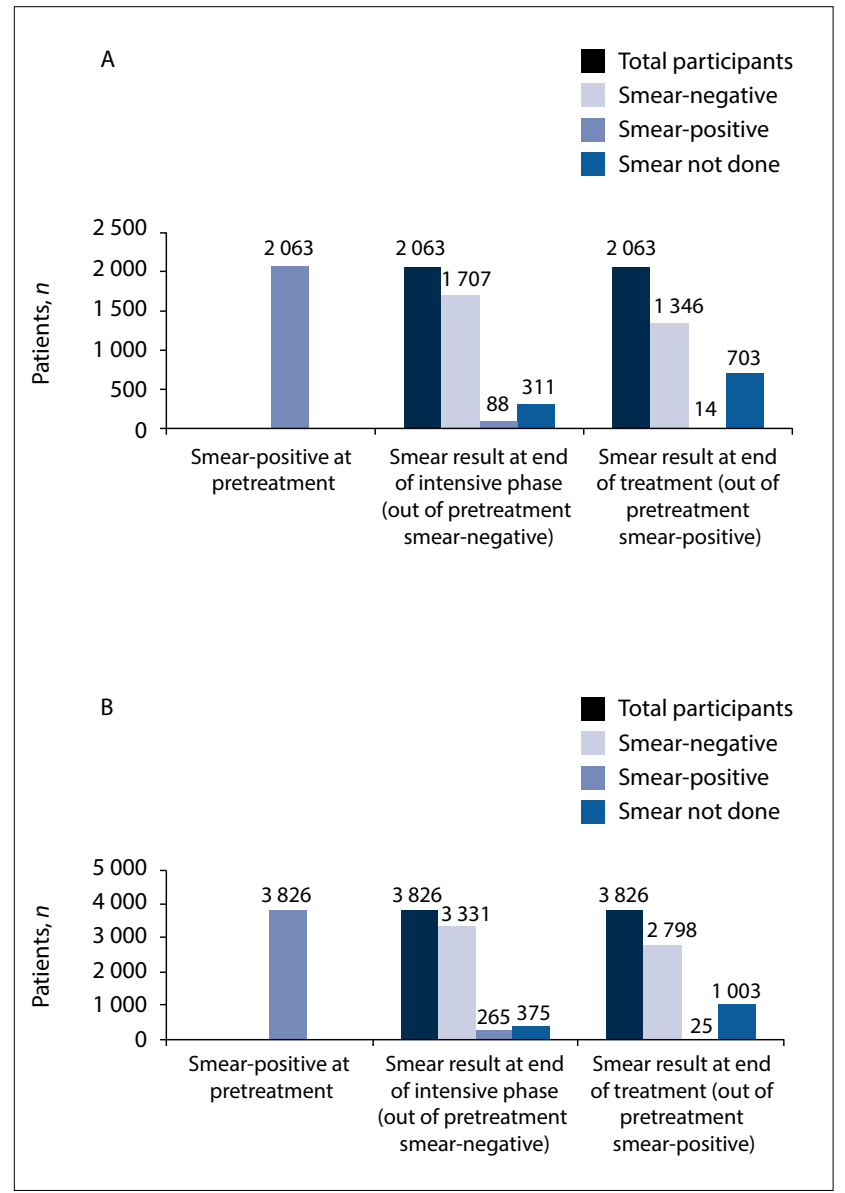

Fig. 2. Diagnostic sputum smear result and conversion at the end of the intensive phase and at the end of treatment in HIV-positive patients ( $A$ ) and HIV-negative patients (B).

\section{Sputum smear microscopy and culture results at diagnosis}

Of 6397 HIV-positive patients, 5466 (85.4\%) had a smear test, and of these only $3559(65.1 \%)$ had smear culture. Of 6275 HIV-negative patients, $5789(92.2 \%)$ had a smear test and of these only 2827 $(48.8 \%)$ had smear culture. Table 5 shows that the sensitivity of the sputum smear in HIV-positive patients was low (47.1\%) compared with that in HIV-negative patients (68.1\%). The specificity of the sputum smear was $9 \%$ higher in HIV-positive patients than in HIVnegative patients.

\section{Risk factors for an unfavourable treatment outcome}

In the multivariate analysis, HIV co-infection (RR 1.2), retreatment (RR 1.4) and smear not done (RR 1.4) were significant risk factors for an unfavourable treatment outcome (Table 6).

Patients in the Eastern (RR 0.9) and Northern (RR 0.7) subdistricts were less likely to have had an unfavourable treatment outcome than those in the Khayelitsha subdistrict.

When stratified by HIV status, retreatment and smear not done were significantly associated with unfavourable treatment outcomes in HIV-negative TB patients, while being from the Northern and Eastern subdistricts compared with Khayelitsha was associated with a favourable outcome.

Co-infected patients aged $<59$ years compared with those aged 15 - 29 years, and co-infected patients who had a negative diagnostic smear recorded compared with those who had a positive smear, were less likely to have an unfavourable outcome. Patients who 
Table 2. Demographic characteristics, HIV status, treatment category, site of $\mathrm{TB}$, subdistrict where treated and diagnostic sputum smear microscopy results of patients initiated on TB treatment in the Metro East GSA, Cape Town, stratified by HIV status

\begin{tabular}{|c|c|c|}
\hline Variables & $\begin{array}{l}\text { HIV-negative } \\
(N=6 \text { 275), } n \text { (\%) }\end{array}$ & $\begin{array}{l}\text { HIV-positive } \\
(N=6397), n(\%)\end{array}$ \\
\hline \multicolumn{3}{|l|}{ Age (years) } \\
\hline $15-29$ & $2606(41.5)$ & $1754(27.4)$ \\
\hline $30-44$ & $1855(29.6)$ & $3588(56.1)$ \\
\hline $45-59$ & $1405(22.4)$ & $967(15.1)$ \\
\hline$>59$ & $409(6.5)$ & $88(1.4)$ \\
\hline \multicolumn{3}{|l|}{ Sex } \\
\hline Female & $2356(37.5)$ & $3410(53.3)$ \\
\hline Male & $3919(62.5)$ & $2987(46.7)$ \\
\hline \multicolumn{3}{|l|}{ Subdistrict } \\
\hline Khayelitsha & $1640(26.1)$ & $3043(47.6)$ \\
\hline Eastern & $1594(25.4)$ & $1347(21.0)$ \\
\hline Northern & 875 (13.9) & $1017(15.9)$ \\
\hline Tygerberg & $2166(34.5)$ & $990(15.5)$ \\
\hline \multicolumn{3}{|l|}{ Outcome } \\
\hline Treatment successful & $4612(73.5)$ & $4258(66.6)$ \\
\hline Treatment unsuccessful & $1489(23.7)$ & $1799(28.1)$ \\
\hline Died & $174(2.8)$ & $340(5.3)$ \\
\hline \multicolumn{3}{|l|}{ Category } \\
\hline New & $4541(72.3)$ & $4275(66.8)$ \\
\hline Retreatment & $1734(27.6)$ & $2122(33.2)$ \\
\hline \multicolumn{3}{|l|}{ Disease classification } \\
\hline Pulmonary & $5575(88.8)$ & $4989(78.0)$ \\
\hline Extrapulmonary & $615(9.8)$ & $1187(18.6)$ \\
\hline Both & $85(1.4)$ & $221(3.5)$ \\
\hline \multicolumn{3}{|l|}{ Smear diagnostic result } \\
\hline Positive & $3826(61.0)$ & $2063(32.2)$ \\
\hline Negative & $1963(31.3)$ & $3403(53.2)$ \\
\hline No smear & $486(7.7)$ & $931(14.6)$ \\
\hline
\end{tabular}

had previously had TB (retreatment cases) were significantly more likely than new patients to have an unfavourable treatment outcome (RRs 1.6 for HIV-negative and 1.3 for co-infected patients).

HIV-negative TB patients from the Eastern and Northern subdistricts were more likely to have a favourable outcome than those from Khayelitsha, while retreatment compared with new patients and those who did not have a smear compared with those who had a positive smear were more likely to have an unfavourable outcome.

\section{Discussion}

Our study showed that the incidence of TB in the Metro East GSA was high at 922 per 100000 . This is alarming. According to the 2014 WHO TB report, the incidence of TB was 125 per 100000 population globally, 165 per 1000000 in 22 high-burden countries and 860 per 100000 in South Africa. ${ }^{[5]}$ The incidence in the Western Cape Province of SA was 885 per 100000 population, ${ }^{[14]}$ and in Cape Town it was 877 per 100000 population in 2009 and 663 per 100000 population in 2013. ${ }^{[15]}$ One of the main reasons for the high incidence is the high prevalence of HIV in some of these areas. ${ }^{[16]}$

In the Metro East GSA of Cape Town, $96.7 \%$ of TB patients had been tested for HIV. This shows that the recommendation that all TB patients be screened for HIV has been successfully implemented. Half of the TB patients in this study were co-infected with HIV. This high co-infection rate was due to the inclusion of the Khayelitsha

\begin{tabular}{|c|c|c|c|}
\hline Variables & $\begin{array}{l}\text { Treatment } \\
\text { successful } \\
(N=8870), \\
n(\%)\end{array}$ & $\begin{array}{l}\text { Treatment } \\
\text { unsuccessful } \\
(N=3288), \\
n(\%) \\
\end{array}$ & $\begin{array}{l}\text { Died } \\
(N=514), \\
n(\%)\end{array}$ \\
\hline \multicolumn{4}{|l|}{ Age (years) } \\
\hline $15-29$ & $3106(35.0)$ & $1176(35.8)$ & $78(15.2)$ \\
\hline $30-44$ & $3754(42.3)$ & $1469(44.7)$ & $220(42.8)$ \\
\hline $45-59$ & $1689(19.0)$ & $530(16.1)$ & $153(39.8)$ \\
\hline$>59$ & $321(3.6)$ & $113(3.4)$ & $63(12.3)$ \\
\hline \multicolumn{4}{|l|}{ Sex } \\
\hline Female & $4068(46.8)$ & $1477(44.9)$ & $221(43.0)$ \\
\hline Male & $4802(54.1)$ & $1811(55.1)$ & $293(57.0)$ \\
\hline \multicolumn{4}{|l|}{ Subdistrict } \\
\hline Khayelitsha & $3056(34.5)$ & $1428(43.4)$ & $199(38.7)$ \\
\hline Eastern & $2128(24.0)$ & $722(22.0)$ & $91(17.7)$ \\
\hline Northern & $1462(16.5)$ & $357(10.9)$ & $73(14.2)$ \\
\hline Tygerberg & $2224(25.1)$ & $781(24.0)$ & $151(29.4)$ \\
\hline \multicolumn{4}{|l|}{ HIV status } \\
\hline Negative & $4612(52.0)$ & $1489(45.3)$ & $174(33.9)$ \\
\hline Positive & $4258(48.0)$ & $1799(54.7)$ & $340(66.1)$ \\
\hline \multicolumn{4}{|l|}{ Category } \\
\hline New & $6460(72.8)$ & $2054(62.5)$ & $302(58.8)$ \\
\hline Retreatment & $2410(27.2)$ & $1234(37.6)$ & $212(41.2)$ \\
\hline \multicolumn{4}{|l|}{$\begin{array}{l}\text { Disease } \\
\text { classification }\end{array}$} \\
\hline Pulmonary & $7454(84.0)$ & $2715(82.6)$ & $395(76.8)$ \\
\hline Extrapulmonary & $1211(13.7)$ & $497(15.1)$ & $94(18.3)$ \\
\hline Both & $205(2.3)$ & $76(2.3)$ & $25(4.9)$ \\
\hline \multicolumn{4}{|l|}{$\begin{array}{l}\text { Smear diagnostic } \\
\text { result }\end{array}$} \\
\hline Positive & $4232(47.7)$ & $1481(45.0)$ & $176(34.2)$ \\
\hline Negative & $3847(43.4)$ & $1264(38.4)$ & 255 (49.6) \\
\hline No smear & $791(8.9)$ & $543(16.6)$ & $83(16.1)$ \\
\hline
\end{tabular}

subdistrict, which has the highest co-infection rate in the Western Cape. ${ }^{[16]}$ Khayelitsha, with the highest antenatal HIV prevalence, had both the highest rate of TB and the highest co-infection rate, while Tygerberg had a comparatively high TB incidence but the lowest prevalence of co-infection. ${ }^{[16]} \mathrm{HIV}$ is therefore not the sole reason for the TB epidemic. Other developing countries such as Pakistan, India and China have a low prevalence of HIV but a high incidence of TB. ${ }^{[17-19]}$

In this study, $45.5 \%$ of $\mathrm{TB}$ patients were female, but more females than males were HIV co-infected (of HIV-positive patients $53.3 \%$ were female, compared with $37.5 \%$ of HIV-negative patients). Co-infected females were also younger than their male counterparts. The epidemic of TB in terms of sex has changed as a result of the HIV epidemic. Before the era of HIV the male-to-female ratio for TB incidence was 2:1, ${ }^{[20]}$ but the high prevalence of HIV in females has led to an increased incidence of TB among females. ${ }^{[21]}$ A number of studies and reports have shown that women of childbearing age were more likely than the older women to develop TB due to co-infection with HIV. ${ }^{[22,23]}$

In this study, $14.2 \%$ of patients with TB had EPTB, and $65.9 \%$ of them were co-infected with HIV. This is similar to other studies that showed that EPTB was more common in co-infected patients. ${ }^{[2,25]}$ 


\begin{tabular}{|c|c|c|c|c|}
\hline & \multicolumn{4}{|c|}{ Subdistrict } \\
\hline & Khayelitsha & Eastern & Northern & Tygerberg \\
\hline TB notification case rate & $\begin{array}{l}4683 / 279212 \\
(1677 / 100000)\end{array}$ & $\begin{array}{l}2941 / 372344 \\
(790 / 100000)\end{array}$ & $\begin{array}{l}1892 / 281691 \\
(672 / 100000)\end{array}$ & $\begin{array}{l}3156 / 441688 \\
(715 / 100000)\end{array}$ \\
\hline $\begin{array}{l}\text { Proportion of TB patients with HIV } \\
\text { co-infection }\end{array}$ & $\begin{array}{l}3043 / 4683 \\
(65.0 \%)\end{array}$ & $\begin{array}{l}1347 / 2941 \\
(45.8 \%)\end{array}$ & $\begin{array}{l}1017 / 1892 \\
(53.8 \%)\end{array}$ & $\begin{array}{l}990 / 3156 \\
(31.4 \%)\end{array}$ \\
\hline HIV prevalence (2012 antenatal survey) & $37.1 \%$ & $17.9 \%$ & $26.2 \%$ & $9.4 \%$ \\
\hline
\end{tabular}

Table 5. Comparison of diagnostic sputum smear results and sputum culture results at pretreatment in HIV-positive and HIV-negative patients

\begin{tabular}{llll}
\hline & \multicolumn{2}{c}{ Culture result } & \\
\cline { 2 - 3 } Sputum result & Positive, $\boldsymbol{n}(\%)$ & Negative, $\boldsymbol{n}(\%)$ & Total \\
\hline HIV-positive & & & \\
$\quad$ Positive & $1216(47.1)$ & $110(11.2)$ & 1326 \\
$\quad$ Negative & $1364(52.9)$ & $869(88.8)$ & 2233 \\
$\quad$ Total & $2580(100)$ & $979(100)$ & 3559 \\
HIV-negative & & & \\
$\quad$ Positive & $1674(68.1)$ & $75(20.2)$ & 1749 \\
$\quad$ Negative & $783(31.9)$ & $295(79.7)$ & 1078 \\
$\quad$ Total & $2457(100)$ & $370(100)$ & 2827 \\
& & &
\end{tabular}

Our study also showed that more retreatment patients were co-infected than new patients. This is similar to another SA study, in which 97\% of retreatment patients were tested for HIV and 78\% were co-infected. ${ }^{[26]} \mathrm{A}$ study from India found that retreatment patients were less likely to be co-infected, but this was in a low HIV prevalence setting. ${ }^{[27]}$

In the Metro East GSA, smear microscopy should not be the only means of diagnosing $\mathrm{TB}$, as $53.2 \%$ of HIV-positive patients and $31.3 \%$ of HIV-negative patients had smear-negative, culture-positive TB. Culture is the gold standard for the diagnosis of TB, but it takes up to 6 weeks and is expensive, so sputum smear microscopy is the cornerstone of the diagnosis in many resource-limited settings. ${ }^{[28,29]}$ Many studies have shown that smear microscopy has low sensitivity. ${ }^{[4,30]}$ Recently GeneXpert, a nucleic acid test that produces results within 2 hours, has been used in the majority of clinics in SA, and this should alleviate the problem of waiting for cultures. ${ }^{[31]}$

Sputum smear microscopy is useful to monitor the progress of TB disease. ${ }^{[27]}$ According to the SA TB guidelines, ${ }^{[28]}$ sputum smear microscopy should be done before treatment is initiated, at the end of the intensive phase (at 2 or 3 months) and at the end of treatment, particularly in patients who had positive bacteriological findings before treatment. At the end of the 3 months $11.6 \%$ of patients with smear-positive TB had not had a smear done, showing that clinics in the Metro East GSA were not following the guidelines. It is possible that these smears were not done because patients were not able to provide a sputum specimen.

Only $70.0 \%$ of TB patients had a successful outcome in the Metro East GSA, far below the target of $85 \%$. This low percentage of successful outcomes may explain why the Western Cape has the highest TB notification rate in the country. ${ }^{[32]}$ The WHO estimates that a treatment completion rate of $85 \%$ and a $70 \%$ case detection rate are required to have an impact on the TB epidemic in high-burden countries. ${ }^{[5]}$ In 2013 , the treatment success rate was $86 \%$ globally. In the same year, the success rate in SA was $77 \%{ }^{[5]}$

The Khayelitsha subdistrict had the highest proportion of unsuccessful treatment outcomes. This could be due to the high burden imposed by TB and HIV co-infection on the healthcare system. Khayelitsha is also one of the poorest areas of Cape Town, with high unemployment and more than $50 \%$ of dwellings being informal. ${ }^{[14,18]}$

HIV co-infection was associated with an unfavourable treatment outcome. This could be due to the negative impact of HIV on TB, increasing the risk of TB-related morbidity and mortality ${ }^{[7]}$ A study from Toronto ${ }^{[33]}$ showed a high treatment failure rate $(36 \%)$ in co-infected patients, and that HIV was an independent risk factor for not completing TB treatment. Another study, from Nigeria, found a treatment failure rate of $27 \%$ in HIV co-infected patients compared with $19 \%$ in HIV-negative patients. ${ }^{[34]}$

Unfavourable outcomes in co-infected patients may be due to a delay in diagnosis because of atypical clinical presentation, and it is also known that co-infected patients tend to have paucibacillary TB or low microbacterial colony counts. ${ }^{[35]}$ This reduction in microbacterial count makes the diagnosis of TB by smear microscopy more difficult.

In contrast to other studies, ${ }^{[36]}$ the present study showed no difference in treatment outcome between the sexes.

Patients who had had TB previously (retreatment cases) were significantly more likely to have an unfavourable $\mathrm{TB}$ treatment outcome than new patients. A number of studies have shown that retreatment patients are at an increased risk of having an unfavourable treatment outcome. ${ }^{[37]}$ Retreatment was more common in co-infected patients, providing an added risk. ${ }^{[38]}$ Furthermore, retreatment patients were more prone to drug-resistant $\mathrm{TB}$ and loss to follow-up, increasing the likelihood of an unfavourable outcome. ${ }^{[2,37]}$

Absence of sputum smear microscopy at diagnosis was also a significant risk factor for an unfavourable treatment outcome. Sputum smear microscopy may not be requested if there is an atypical clinical presentation of TB, such as absence of a cough and inability to produce sputum. Absence of sputum smear microscopy at diagnosis may also be due to health system-related failures. Most clinics do not have a physiotherapist to assist patients who are finding it difficult to produce sputum, sputum may not be requested owing to lack of resources or tardiness on the part of clinical staff, or the results may not be received by the clinic, or be received but not entered in the patient's record.

\section{Study strengths and limitations}

The major strength of this study was the large database from an area with a high incidence of TB and a varying prevalence of HIV, showing the impact of HIV together with other factors. HIV status was confirmed from clinical records rather than self-reported, and the accuracy of the data was therefore likely to be good. Only $2.8 \%$ of patients were excluded because they did not have an HIV test result.

A major limitation was missing or incomplete data, and this may have affected the power of the study. It was a secondary analysis of data already collected, and the principal investigator (SM) was not 
Table 6. Risk factors for unfavourable treatment outcome, stratified by HIV status

\begin{tabular}{|c|c|c|c|c|c|c|c|c|}
\hline \multirow[b]{2}{*}{ Variables } & \multicolumn{2}{|c|}{$\begin{array}{l}\text { Univariate analysis, } \\
\text { whole population } \\
(N=12672,100 \%)\end{array}$} & \multicolumn{2}{|c|}{$\begin{array}{l}\text { Multivariate analysis, } \\
\text { whole population } \\
(N=12672,100 \%)\end{array}$} & \multicolumn{2}{|c|}{$\begin{array}{l}\text { HIV-negative } \\
(n=6275,49.5 \%)\end{array}$} & \multicolumn{2}{|c|}{$\begin{array}{l}\text { HIV-positive } \\
(n=6397,50.5 \%)\end{array}$} \\
\hline & RR ( $95 \% \mathrm{CI})$ & $p$-value & $\mathrm{RR}(95 \% \mathrm{CI})$ & $p$-value & RR $(95 \% \mathrm{CI})$ & $p$-value & RR (95\% CI) & $p$-value \\
\hline \multicolumn{9}{|l|}{ HIV status } \\
\hline Negative & Ref & & Ref & & & & & \\
\hline Positive & $1.3(1.2-1.3)$ & 0.000 & $1.2(1.1-1.3)$ & 0.000 & & & & \\
\hline \multicolumn{9}{|l|}{ Age (years) } \\
\hline $15-29$ & Ref & & Ref & & Ref & & Ref & \\
\hline $30-44$ & $1.1(1.0-1.2)$ & 0.015 & $1.0(0.9-1.0)$ & 0.173 & $1.0(0.9-1.1)$ & 0.569 & $0.9(0.8-0.9)$ & 0.018 \\
\hline $45-59$ & $1.0(0.9-1.1)$ & 0.978 & $0.9(0.8-1.0)$ & 0.012 & $0.9(0.8-1.0)$ & 0.090 & $0.9(0.8-0.9)$ & 0.020 \\
\hline$>59$ & $1.2(1.1-1.4)$ & 0.001 & $1.1(1.0-1.3)$ & 0.030 & $1.1(1.0-1.3)$ & 0.138 & $1.1(0.9-1.4)$ & 0.235 \\
\hline \multicolumn{9}{|l|}{ Sex } \\
\hline Female & Ref & & Ref & & Ref & & Ref & \\
\hline Male & $1.0(1.0-1.1)$ & 0.214 & $1.1(1.0-1.1)$ & 0.005 & $1.1(1.0-1.3)$ & 0.286 & $1.1(1.0-1.2)$ & 0.007 \\
\hline \multicolumn{9}{|l|}{ Category } \\
\hline New & Ref & & Ref & & Ref & & Ref & \\
\hline Retreatment & $1.4(1.3-1.5)$ & 0.000 & $1.4(1.3-1.5)$ & 0.000 & $1.6(1.5-1.7)$ & 0.000 & $1.3(1.2-1.4)$ & 0.000 \\
\hline \multicolumn{9}{|l|}{$\begin{array}{l}\text { Sputum smear } \\
\text { diagnostic result }\end{array}$} \\
\hline Positive & Ref & & Ref & & Ref & & Ref & \\
\hline Negative & $1.0(0.9-1.1)$ & 0.843 & $0.9(0.9-1.0)$ & 0.047 & $0.9(0.8-1.1)$ & 0.410 & $0.9(0.8-0.9)$ & 0.037 \\
\hline Smear not done & $1.6(1.5-1.7)$ & 0.000 & $1.4(1.3-1.5)$ & 0.000 & $1.6(1.4-1.7)$ & 0.000 & $1.3(1.2-1.5)$ & 0.000 \\
\hline \multicolumn{9}{|l|}{ Subdistrict } \\
\hline Khayelitsha & Ref & & Ref & & Ref & & Ref & \\
\hline Eastern & $0.8(0.7-0.9)$ & 0.000 & $0.9(0.8-0.9)$ & 0.000 & $0.9(0.8-0.9)$ & 0.005 & $0.8(0.7-0.9)$ & 0.000 \\
\hline Northern & $0.6(0.6-0.7)$ & 0.000 & $0.7(0.7-0.8)$ & 0.000 & $0.7(0.6-0.8)$ & 0.000 & $0.7(0.6-0.8)$ & 0.000 \\
\hline Tygerberg & $0.8(0.8-0.9)$ & 0.000 & $0.9(0.9-1.0)$ & 0.042 & $0.9(0.9-1.0)$ & 0.004 & $1.0(0.9-1.1)$ & 0.926 \\
\hline \multirow{2}{*}{\multicolumn{9}{|c|}{$\begin{array}{l}\text { Disease } \\
\text { classification }\end{array}$}} \\
\hline & & & & & & & & \\
\hline Pulmonary & Ref & & Ref & & Ref & & Ref & \\
\hline Extrapulmonary & $1.1(1.0-1.2)$ & 0.003 & - & & - & & - & \\
\hline Both & $1.1(1.0-1.3)$ & 0.157 & - & & - & & - & \\
\hline $\mathrm{CI}=$ confidence interval. & & & & & & & & \\
\hline
\end{tabular}

able to trace missing information from the primary clinical records. In addition, there may have been recording and data-capturing errors that the principal investigator was not able to correct. Moreover, limited information was collected on patients on ETR.Net and we could therefore not adjust for known confounders such as socioeconomic status, education level and marital status. All patients with an unrecorded treatment outcome were regarded as having an unfavourable treatment outcome. Patients with unrecorded HIV status were excluded, and it is not known whether this group would have been more or less likely to be co-infected.

\section{Conclusions}

The incidence of TB is extremely high in the Metro East GSA of Cape Town, but the prevalence of co-infection with HIV varies across the subdistricts. Co-infection, retreatment and smear microscopy not done before treatment were factors significantly associated with an unfavourable treatment outcome. The Eastern and Northern subdistricts were significantly more likely to have favourable treatment outcomes compared with Khayelitsha, where both TB incidence and HIV co-infection were highest. More research is required to identify other risk factors for unfavourable treatment outcomes.
Acknowledgements. SM thanks Katherine Hilderbrand, of the Department of Public Health, UCT, for giving great insight into the tuberculosis electronic register, and Henri Carrara, of the same department, for muchneeded hints and suggestions on the statistical analyses.

Author contributions. SM (principal investigator): study conception and design, literature review, drafting of manuscript, analysis and interpretation of data; DC: study conception and design, critical revision, acquisition of data.

Funding. None.

Conflicts of interest. None.

1. Mukadi YD, Maher D, Harries A. Tuberculosis case fatality rates in high HIV prevalence populations in sub-Saharan Africa. AIDS 2001;15(2):143-152. https://dx.doi.org/10.1097/00002030-200101260-00002 Malkin JE, Prazuck T, Simonet F, et al. Tuberculosis and human immunodeficiency virus infection in West Burkina Faso: Clinical presentation and clinical evolution. Int J Tuberc Lung Dis 1997;1(1):68-74. 3. Banerjee A, Moyo S, Salaniponi F, Harries A. HIV testing and tuberculosis outcome in a rural district in Malawi. Trans R Soc Trop Med Hyg 1997;91(6):707-708. https://dx.doi.org/10.1016/s00359203(97)90533-2

4. Cunningham J, Perkins M. Diagnostics for tuberculosis: Global demand and market potential. 2006. http://popline.org/node/181705 (accessed 20 September 2016).

5. World Health Organization. Global Tuberculosis Report, 2014. Geneva: WHO, 2014.

6. Wood R, Lawn SD, Caldwell J, Kaplan R, Middelkoop K, Bekker L. Burden of new and 6. Wood R, Lawn SD, Caldwell J, Kaplan R, Middelkoop K, Bekker L. Burden of new and
recurrent tuberculosis in a major South African city stratified by age and HIV-status. PLoS One 2011;6(10):e25098. https://dx.doi.org/10.1371/journal.pone.0025098

7. Lucas S, Nelson AM. Pathogenesis of tuberculosis in human immunodeficiency virus-infected people. In: Bloom B, ed. Tuberculosis: Pathogenesis, Protection, and Control. Washington, DC: ASM Press,
Ins 1994:503-513. https://dx.doi.org/10.1128/9781555818357.ch29 
8. Toossi Z, Mayanja-Kizza H, Hirsch C, et al. Impact of tuberculosis (TB) on HIV-1 activity in dually infected patients. Clin Exp Immunol 2001;123(2):233-238. https://dx.doi.org/10.1046/.13652249.2001.01401.x

9. Statistics South Africa. Mortality and causes of death in South Africa 2010: Findings from death notification. 2013. www.statssa.gov.za/publications/P03093/P030932010.pdf (accessed 27 March
notich 2016).

10. World Health Organization. Tuberculosis Country Profile: South Africa, 2013. Geneva: WHO, 2014. http://who.int/tb/country/data/profiles/en/ (accessed 27 March 2016)

11. World Health Organization. Global Tuberculosis Report, 2013. Geneva: WHO, 2013.

12. Statistics South Africa. Census 2011. Pretoria: SSA, 2012. https://www.statssa.gov.za/publications/ P03014/P030142011.pdf (accessed 31 March 2013).

13. National Department of Health, South Africa. The 2012 National Antenatal Sentinel HIV \& Herpes Simplex Type-2 Prevalence Survey in South Africa. Pretoria: NDoH, 2013. http://aviwe.wrhi.ac.za/ ashivherp_report2014_22may2014/ (accessed 31 March 2017).

14. Western Cape Health Department. Provincial strategy plan on HIV/AIDS, STI and TB, 2012 - 2016. 2012. https://www.westerncape.gov.za/assets/departments/health/provincial_strategic_plan_on_hiv aids_stis_tb_2012_-_2016_-_15_june_2012.pdf (accessed 23 November 2015).

15. Osman M, Seddon JA, Dunbar R, Draper HR, Lombard C, Beyers N. The complex relationship between human immunodeficiency virus infection and death in adults being treated for tuberculosis in Cape Town, South Africa. BMC Public Health 2015;15:556. http://dx.doi.org/10.1186/s12889-015-1914-z

16. Draper B, Pienaar D, Parker W, Rehle T. Recommendations for policy in the Western Cape province for the prevention of major infectious diseases, including HIV/AIDS and tuberculosis. Cape Town, 2007. the prevention of major infectious diseases, including HIV/AIDS and tuberculosis. Cape Town, 2007.

17. Siddiqui M, Fakih A Burney W Iftikhar $R$, Khan N. Environmental and host related factors predisposing to tuberculosis in Karachi: A cross-sectional study J Pak Med Stud 2011:1(1):13-18.

18. Shetty N, Shemko M, Vaz M, D'Souza G. An epsidemiological evaluation of risk factors for tuberculosis in South India: A matched case control study. Int J Tuberc Lung Dis 2006;10(1):80-86.

19. Chen W, Shu W, Wang M, et al. Pulmonary tuberculosis incidence and risk factors in rural areas of 9. Chen W, Shu W, Wang M, et al. Pulmonary tuberculosis incidence and risk factors in rural areas of
China: A cohort study. PloS One 2013;8(3):e58171. https://dx.doi.org/10.1371/journal.pone.0058171 20. Rao S. Tuberculosis and patient gender: An analysis and its implications in tuberculosis control. Lung India 2009;26(2):46-47. https://dx.doi.org/10.4103/0970-2113.48897

21. Lawn SD, Bekker LG, Middelkoop K, Myer L, Wood R. Impact of HIV infection on the epidemiology of tuberculosis in a peri-urban community in South Africa: The need for age-specific interventions. Clin Infect Dis 2006;42(7):1040-1047. https://dx.doi.org/10.1086/501018

22. World Health Organization. Tuberculosis in women: Tuberculosis fact sheet 2014. http://www.who. int/tb/publications/tb_women_factsheet_251013.pdf (accessed 3 November 2015).

23. Perumal R, Padayatchi N, Naidoo K, Knight $\mathrm{S}$. Understanding the profile of tuberculosis and human immunodeficiency virus coinfection: Insights from expanded HIV surveillance at a tuberculosis facility in Durban, South Africa. ISRN AIDS 2014;2014:260329. https://dx.doi.org/10.1155/2014/260329

24. Raviglione MC, Narain JP, Kochi A. HIV-associated tuberculosis in developing countries: Clinical features, diagnosis, and treatment. Bull World Health Organ 1992;70(4):515-526. https://dx.doi. org/10.1016/0962-8479(92)90033-g
25. Karstaedt AS. Extrapulmonary tuberculosis among adults: Experience at Chris Hani Baragwanath Academic Hospital, Johannesburg, South Africa. S Afr Med J 2014;104(1):22-24. https://dx.doi. org/10.7196/SAMJ.6374

26. Middelkoop K, Bekker LG, Shashkina E, Kreiswirth B, Wood R. Retreatment tuberculosis in a South African community: The role of re-infection, HIV and antiretroviral treatment. Int J Tuberc Lung Dis 2012:16(11):1510-1516. https://dx.doi.org/10.5588/ijtld.12.0049

27. Shastri S, Naik B, Shet A, Rewari B, De Costa A. TB treatment outcomes among TB-HIV co-infection in Karnataka, India: How do these compare with non-HIV tuberculosis outcomes in the province? BMC Public Health 2013;13(1):838. https://dx.doi.org/10.1186/1471-2458-13-838

28. National Department of Health, South Africa. National Tuberculosis Management Guidelines 2014 2014. http://www.tbonline.info/media/uploads/documents/ntcp_adult_tb-guidelines-27.5.2014.pd (accessed 7 October 2014)

29. Ozkutuk A, Terek G, Coban H, Esen N. Is it valuable to examine more than one sputum smear pe patient for the diagnosis of pulmonary tuberculosis? Jpn J Infect Dis 2007;60(2/3):73.

30. Steingart KR, $\mathrm{Ng} \mathrm{V}$, Henry M, et al. Sputum processing methods to improve the sensitivity of smear microscopy for tuberculosis: A systematic review. Lancet Infect Dis 2006;6(10):664-674. https://dx.doi org/10.1016/s1473-3099(06)70602-8

31. TB fact.org. Xpert test for TB diagnosis: TB fact.org. http://www.tbfacts.org/xpert-tb-test.htm (accessed 28 November 2015).

32. Den Boon S, van Lill SW, Borgdorff MW, et al. High prevalence of tuberculosis in previously treated patients, Cape Town, South Africa. Emerg Infect Dis 2007;13(8):1189-1194. https://dx.do org/10.3201/eid 1308.051327

33. Wobeser W, Yuan L, Naus M. Outcome of pulmonary tuberculosis treatment in the tertiary care setting - Toronto 1992/93. Tuberculosis Treatment Completion Study Group. CMAJ 1999;160(6):789-794.

34. Jibrin YB, Ali AB, Saad ST, Kolo PM. Prevalence of treatment failure among pulmonary tuberculosis patients in Federal Medical Centre, Gombe, northeastern Nigeria. ISRN Infect Dis 2013;2013:46104. https://dx.doi.org/10.5402/2013/461704

35. Aderaye G, Bruchfeld J, Assefa G, et al. The relationship between disease pattern and disease burden by chest radiography $M$. tuberculosis load, and HIV status in patients with pulmonary tuberculosis in Addis Ababa. Infection 2004;32(6):333-338. https://dx.doi.org/10.1007/s15010-004-3089-x

36. Sen G, Ostlin P, George A. Gender inequity in health: Why it exists and how we can change it. Glob Public Health 2008;3(1):1-12. https://dx.doi.org/10.1080/17441690801900795

37. Kritski AL, de Jesus LSR, Werneck-Barroso E, et al. Retreatment tuberculosis cases: Factors associated . chest.111.5.1162

38. Chaisson RE, Churchyard GJ. Recurrent tuberculosis: Relapse, reinfection, and HIV. J Infect Dis 2010;201(5):653-655. https://dx.doi.org/10.1086/650531

Accepted 26 January 2017. 